\title{
Stability Properties of Linear Volterra Discrete Systems With Nonlinear Perturbation
}

\section{TOUHID M. KHANDAKER AND YOUSSEF N. RAFFOUL}

Department of Mathematics, University of Dayton, Dayton, OH 45469-2316

Dedicated to Allan Peterson on the occasion of his 60th birthday.

We consider a Volterra discrete system with nonlinear perturbation

$$
x(n+1)=A(n) x(n)+\sum_{s=0}^{n} B(n, s) x(s)+g(n, x(n))
$$

and obtain necessary and sufficient conditions for stability properties of the zero

solution employing the resolvent equation coupled with the variation of parameters formula.

Keywords: Resolvent; Nonlinear perturbation; Variation of parameters; Uniform stability; Uniform asymptotic stability; Liapunov functionals

Classification Categories: 34D20,39A10,39A12,40A05

\section{INTRODUCTION}

In this paper, we have studied the stability properties of the zero solution of the nonlinear perturbed volterra discrete system

$$
x(n+1)=A(n) x(n)+\sum_{s=0}^{n} B(n, s) x(s)+g(n, x(n))
$$

where $g(n, x(n))$ is continuous in $x$ and satisfies $|g(n, x(n))| \leq \lambda(n)|x(n)|$, where $\lambda(n)$ is such that $0 \leq \lambda(n) \leq N<+\infty$, for some constant $N$.

Moreover, $A(n), B(n, s)$ are $n \times n$ matrix functions on $\mathbb{Z}^{+}$and $\mathbb{Z}^{+} \times \mathbb{Z}^{+}$, respectively. Recently, several authors have studied the behavior of solutions of variant forms of (1.1). Medina [10], [11], [12], Eloe, Islam and Raffoul [5], and Raffoul [13], obtained stability and boundedness results of the solutions 
of the homogenous part of (1.1) by means of representing the solution in terms of the resolvent matrix. Eloe et al. [5] and Elaydi et al. [4], used the notion of total stability and established results on the asymptotic behavior of the zero solution of (1.1). Their work heavily depended on showing or assuming the summability of the resolvent matrix. For more results on stability of the zero solution of Volterra discrete system we refer the reader to Crisci, Komanovskii and Vecchio [2], Elaydi [3] and Agarwal and Pang [1]. This research is a continuation of the research initiated by the authors in [6] and related to the work in [5]. In this paper we extend some of the results in [7], and later in the paper, we furnish an example as an application to some of our theorems, in which we show the summability of the resolvent matrix.

For $x \in \mathbb{R}^{k},|x|$ denotes the Euclidean norm of $x$. For any $k \times k$ matrix $A$, we define the norm of $A$ by $|A|=\max \{|A x|:|x| \leq 1\}$. We define the set $C(n)=\left\{\phi \in \mathbb{R}^{k}: \phi:[0, n] \rightarrow \mathbb{R}^{k}\right\}$ with the norm $\|\phi\|=\max \{|\phi(s)|: 0 \leq$ $s \leq n\}$ on it.

For each $n_{0} \in \mathbb{Z}^{+}$, and $\phi \in C\left(n_{0}\right)$, there is a unique (vector) function $\phi: \mathbb{Z}^{+} \rightarrow \mathbb{R}^{k}$ on $0 \leq s \leq n_{0}$ on $\left[n_{0}, \infty\right)$ with $x(s)=\phi(s)$ for $0 \leq s \leq$ $n_{0}$. Such a function $x(n)$ is called a solution of (1.1), and is denoted by $x\left(n, n_{0}, \phi\right)$. Throughout the paper we write $x(n)$ for $x\left(n, n_{0}, \phi\right)$ unless it is stated otherwise.

We have also used the resolvent of (1.1) in the analysis. In particular, we have obtained various conditions for the stability properties of the zero solution of (1.1). The resolvent matrix $R(n, s)$ associated with the homogeneous equation of $(1.1)$

$$
x(n+1)=A(n) x(n)+\sum_{s=0}^{n} B(n, s) x(s)
$$

satisfies the resolvent matrix equation

$$
R(n+1, s)=A(n) R(n, s)+\sum_{u=s}^{n} B(n, u) R(u, s)
$$

if $s \leq n, R(s, s)=I$ and $R(n, s)=0$ if $n<s$, or,

$$
R(n, s+1)(A(s)-I)+\sum_{u=s}^{n-1} R(n, u+1) B(u, s)+\Delta_{s} R(n, s)=0
$$

if $s \leq n, R(n, n)=I$ and $R(n, s)=0$ if $n<s$, where $\Delta_{s} R(n, s)=R(n, s+$ 1) $-R(n, s)$. 
Recently, Eloe, Islam and Raffoul arrived at the resolvent equation (1.4) and therefore, for its proof we refer the interested reader to [5].

\section{STABILITY}

Definition 2.1. The zero solution of (1.1) is stable if for each $\epsilon>0$ and $n_{0} \geq 0$, there exists $\delta>0$ such that $\left[\phi \in C\left(n_{0}\right),\|\phi\| \leq \delta, n \geq n_{0}\right]$ implies $|x(n)|<\epsilon$. It is uniformly stable (US) if $\delta$ is independent of $n_{0}$.

Definition 2.2. The zero solution of (1.1) is uniformly asymptotically stable (UAS) if it is US and there exists a $\delta_{0}>0$ with the property that for each $\epsilon>0$ there exists a $T=T(\epsilon)>0$ such that $\left[n_{0}>0, \phi \in C\left(n_{0}\right),\|\phi\|<\right.$ $\delta_{0}$ and $\left.n \geq n_{0}+T\right]$ imply $\left|x\left(n, n_{0}, \phi\right)\right|<\epsilon$.

THEOREM 2.1. Let

$$
Q(n)=|A(n)|+\lambda(n)+\sum_{u=n}^{\infty}|B(u, n)| .
$$

Suppose that, for all $n \geq n_{0} \geq 0$,

$$
\sum_{n=n_{0}}^{\infty} Q(n)\left|R\left(n, n_{0}\right)\right|<\infty, \quad \sum_{u=n}^{\infty} Q(u)|R(u, n+1)| \leq K_{1} Q(n)<\infty
$$

and for the upper bound $N$, on $\lambda(n), 0 \leq N K_{1}<1$, hold. Then the zero solution of $(1.1)$ is stable.

Proof. Suppose that $x(n)$ is a solution of (1.1). If $R(n, s)$ satisfies (1.4), then $x(n)$ is given by, see [5],

$$
\begin{aligned}
x(n)= & R\left(n, n_{0}\right) \phi\left(n_{0}\right)+\sum_{s=n_{0}}^{n-1} R(n, s+1) \sum_{u=0}^{n_{0}-1} B(s, u) \phi(u) \\
& +\sum_{s=n_{0}}^{n-1} R(n, s+1) g(s, x(s)) .
\end{aligned}
$$

First we take the absolute value on both sides of (2.2), multiply through by $Q(n)$ and then sum from $n=n_{0}$ to $n=\infty$ to obtain 


$$
\begin{aligned}
\sum_{n=n_{0}}^{\infty} Q(n)|x(n)| \leq & \sum_{n=n_{0}}^{\infty} Q(n)\left|R\left(n, n_{0}\right)\right|\|\phi\| \\
& +\sum_{n=n_{0}}^{\infty} Q(n) \sum_{s=n_{0}}^{n-1}|R(n, s+1)| \sum_{u=0}^{n_{0}-1}|B(s, u)|\|\phi\| \\
& +\sum_{n=n_{0}}^{\infty} Q(n) \sum_{s=n_{0}}^{n-1}|R(n, s+1)| \lambda(s)|x(s)| \\
\leq & \sum_{n=n_{0}}^{\infty} Q(n)\left|R\left(n, n_{0}\right)\right||| \phi|| \\
& +\sum_{n=n_{0}}^{\infty} \sum_{s=n_{0}}^{n} Q(n)|R(n, s+1)| \sum_{u=0}^{n_{0}}|B(s, u)|\|\phi\| \\
& +\sum_{n=n_{0}}^{\infty} \sum_{s=n_{0}}^{n} Q(n)|R(n, s+1)| \lambda(s)|x(s)| .
\end{aligned}
$$

By changing the order of summations, we have

$$
\sum_{n=n_{0}}^{\infty} \sum_{s=n_{0}}^{n} Q(n)|R(n, s+1)|=\sum_{s=n_{0}}^{\infty} \sum_{n=s}^{\infty} Q(n)|R(n, s+1)| .
$$

Thus, from the above inequality, we obtain

$$
\begin{aligned}
\sum_{n=n_{0}}^{\infty} Q(n)|x(n)| \leq & \sum_{n=n_{0}}^{\infty}\left|R\left(n, n_{0}\right)\right| Q(n)\|\phi\| \\
& +\sum_{s=n_{0}}^{\infty} \sum_{n=s}^{\infty} Q(n)|R(n, s+1)| \sum_{u=0}^{n_{0}}|B(s, u)|\|\phi\| \\
& +\sum_{n=n_{0}}^{\infty} \sum_{u=n}^{\infty} Q(u)|R(u, n+1)| \lambda(n)|x(n)| .
\end{aligned}
$$

By (2.1) there exists an $M_{1}>0$, and $M_{2}>0$ such that

$$
\sum_{n=s}^{\infty} Q(n)|R(n, s)|<M_{1} \text {, and } \sum_{s=n}^{\infty} Q(s)|R(s, n+1)|<M_{2} .
$$


Let

$$
C^{*}\left(n_{0}\right)=\sum_{s=n_{0}}^{\infty} \sum_{u=0}^{n_{0}}|B(s, u)| .
$$

Then from (2.3), we have

$$
\begin{aligned}
\sum_{n=n_{0}}^{\infty} Q(n)|x(n)| \leq & M_{1}\|\phi\|+M_{2} \sum_{s=n_{0}}^{\infty} \sum_{u=0}^{n_{0}}|B(s, u)|\|\phi\| \\
& +N K_{1} \sum_{n=n_{0}}^{\infty} Q(n)|x(n)| \\
\leq & M_{1}\|\phi\|+M_{2} C^{*}\left(n_{0}\right)\|\phi\| \\
& +N K_{1} \sum_{n=n_{0}}^{\infty} Q(n)|x(n)| .
\end{aligned}
$$

Solving for $\sum_{n=n_{0}}^{\infty} Q(n)|x(n)|$, we get

$$
\sum_{n=n_{0}}^{\infty} Q(n)|x(n)| \leq \frac{\|\phi\|\left(M_{1}+M_{2} C^{*}\left(n_{0}\right)\right)}{1-N K_{1}} .
$$

Next we rewrite (1.1) as

$$
\Delta x(n)=D(n) x(n)+\sum_{s=0}^{n} B(s, u) x(s)+g(n, x(n))
$$

where $I$ is the identity matrix and $D(n)=A(n)-I$. By summing the above equation over $n$ from $n_{0}$ to $n-1$ we get

$$
\begin{aligned}
\left|\sum_{s=n_{0}}^{n-1} \Delta x(s)\right|= & \left|\sum_{s=n_{0}}^{n-1}\left[D(s) x(s)+\sum_{u=0}^{s} B(s, u) x(u)+g(s, x(s))\right]\right| \\
\leq & \left|\sum_{s=n_{0}}^{n-1}\left[D(s) x(s)+\sum_{u=n_{0}+1}^{s} B(s, u) x(u)+g(s, x(s))\right]\right| \\
& +\left|\sum_{s=n_{0}}^{n-1} \sum_{u=0}^{n_{0}} B(s, u) x(u)\right| \\
\leq & \sum_{n=n_{0}}^{\infty}|D(n)||x(n)|+\sum_{n=n_{0}}^{\infty} \sum_{u=n_{0}}^{n}|B(n, u)||x(n)| \\
& +\sum_{n=n_{0}}^{\infty} \lambda(n)|x(n)|+\sum_{n=n_{0}}^{\infty} \sum_{u=0}^{n_{0}}|B(n, u)||| \phi|| .
\end{aligned}
$$


By interchanging the order of summations in the second term of the right side of the above inequality, we arrive at

$$
\begin{aligned}
\left|\sum_{s=n_{0}}^{n-1} \Delta x(s)\right| & \leq \sum_{n=n_{0}}^{\infty}\left[|D(n)|+\sum_{u=n_{0}}^{n}|B(n, u)|+\lambda(n)\right]|x(n)|+C^{*}\left(n_{0}\right)\|\phi\| \\
& \leq \sum_{n=n_{0}}^{\infty} Q(n)|x(n)|+C^{*}\left(n_{0}\right)\|\phi\|
\end{aligned}
$$

By substituting (2.4) into (2.5), we get

$$
\left|\sum_{s=n_{0}}^{n-1} \Delta x(s)\right| \leq \frac{\|\phi\|\left(M_{1}+M_{2} C^{*}\left(n_{0}\right)\right)}{1-N K_{1}}+C^{*}\left(n_{0}\right)\|\phi\| .
$$

Thus,

$$
\left|x(n)-x\left(n_{0}\right)\right| \leq\|\phi\|\left[\frac{\|\phi\|\left(M_{1}+M_{2} C^{*}\left(n_{0}\right)\right)}{1-N K_{1}}+C^{*}\left(n_{0}\right)\right] .
$$

But $|x(n)|-\left|x\left(n_{0}\right)\right| \leq\left|x(n)-x\left(n_{0}\right)\right|$, and hence we have

$$
|x(n)| \leq\|\phi\|\left[\frac{\left(M_{1}+M_{2} C^{*}\left(n_{0}\right)\right)}{1-N K_{1}}+C^{*}\left(n_{0}\right)+1\right] .
$$

This completes the proof. We remark that if $C^{*}\left(n_{0}\right)$ is uniformly bounded, then Theorem 2.1 implies that the zero solution of (1.1) is US.

Theorem 2.2. Suppose i.

$$
\begin{gathered}
\sup _{n \geq n_{0} \geq 0} \sum_{s=n}^{\infty}\left|R\left(s, n_{0}\right)\right|<+\infty, \\
\sup _{n \geq n_{0} \geq 0}\left\{\left|R\left(n, n_{0}\right)\right|+\sum_{u=0}^{n_{0}-1} \mid \sum_{s=n_{0}}^{n-1} R(n, s+1) B(s, u)\right\}<+\infty, \\
\sup _{n \geq n_{0} \geq 0} \lambda(n) \sum_{s=n}^{\infty}|R(s, n+1)| \leq L<1,
\end{gathered}
$$

and there exist a $D>0$ such that

$$
\sum_{s=n_{0}}^{\infty}\left(\sum_{n=s}^{\infty}|R(n, s+1)| \sum_{u=0}^{n_{0}-1}|B(s, u)|\right) \leq D
$$


then the zero solution of (1.1) is uniformly stable. ii. If the zero solution of (1.1) is uniformly stable and

$$
\sup _{n \geq n_{0} \geq 0} \sum_{s=n_{0}}^{n-1}|R(n, s+1)|<\infty
$$

then (2.7) holds.

Proof.

i. Suppose that (2.7) - (2.9) hold. Summing (2.2) over $n$ from $n=n_{0}$ to $n=\infty$ and using $|g| \leq \lambda|x|$, we get

$$
\begin{aligned}
\sum_{n=n_{0}}^{\infty}|x(n)| \leq & \sum_{n=n_{0}}^{\infty}\left|R\left(n, n_{0}\right)\right| \| \phi|| \\
& +\sum_{n=n_{0}}^{\infty}\left(\sum_{s=n_{0}}^{n-1}|R(n, s+1)| \sum_{u=0}^{n_{0}-1} B(s, u)\|\phi\|\right) \\
& +\sum_{n=n_{0}}^{\infty}\left(\sum_{s=n_{0}}^{n}|R(n, s+1)||g(s, x(s))|\right) \\
\leq & \sum_{n=n_{0}}^{\infty}\left|R\left(n, n_{0}\right)\right||| \phi|| \\
& +\sum_{n=n_{0}}^{\infty}\left(\sum_{s=n_{0}}^{n}|R(n, s+1)| \sum_{u=0}^{n_{0}-1} B(s, u)\|\phi\|\right) \\
& +\sum_{n=n_{0}}^{\infty} \sum_{s=n}^{\infty}|R(s, n+1)| \lambda(n)|x(n)| .
\end{aligned}
$$

From (2.6), there exists a positive constant $F$ such that $\sum_{s=n}^{\infty}\left|R\left(n, n_{0}\right)\right| \leq F$. Thus, using (2.8) and (2.9) we arrive at

$$
\begin{aligned}
\sum_{n=n_{0}}^{\infty}|x(n)| \leq & F+\sum_{s=n_{0}}^{\infty}\left(\sum_{n=s}^{\infty}|R(n, s+1)| \sum_{u=0}^{n_{0}-1} B(s, u)\right)\|\phi\| \\
& +L \sum_{n=n_{0}}^{\infty} \mid x(n) \| .
\end{aligned}
$$

Hence (2.11) yields

$$
\sum_{n=n_{0}}^{\infty}|x(n)| \leq F\|\phi\|+D\|\phi\|+L \sum_{n=n_{0}}^{\infty}|x(n)| .
$$


Thus,

$$
\sum_{n=n_{0}}^{\infty}|x(n)| \leq \frac{(F+D)\|\phi\|}{1-L} .
$$

Using equation (2.2), we obtain

$$
\begin{aligned}
|x(n)| \leq & \left|R\left(n, n_{0}\right)\right|\|\phi\|+\sum_{u=0}^{n_{0}-1}\left(\left|\sum_{s=n_{0}}^{n-1} R(n, s+1) B(s, u)\right|\right)\|\phi\| \\
& +\sum_{s=n_{0}}^{n-1}|R(n, s+1)| \lambda(s)|x(s)| .
\end{aligned}
$$

By (2.7) and the fact that $\lambda(n)$ is bounded, there exists a constant $P>0$ such that $|R(n, s)| \lambda(s) \leq P$ for $0 \leq n_{0} \leq s \leq n$. Also by (2.7), there exists a constant $E>0$ such that

$$
\sup _{n \geq n_{0} \geq 0}\left\{\left|R\left(n, n_{0}\right)\right|+\sum_{u=0}^{n_{0}-1}\left|\sum_{s=u_{0}}^{n-1} R(n, s+1) B(s, n)\right|\right\}<E .
$$

Thus (2.12) and (2.13) yield

$$
\begin{aligned}
|x(n)| \leq & \left\{\left|R\left(n, n_{0}\right)\right|+\sum_{u=0}^{n_{0}-1}\left|\sum_{s=n_{0}}^{n-1} R(n, s+1) B(s, u)\right|\right\}\|\phi\| \\
& +P \sum_{s=n_{0}}^{n-1}|x(s)| \\
\leq & E\|\phi\|+P \frac{(F+D)\|\phi\|}{1-L}:=J\|\phi\|
\end{aligned}
$$

Thus, (2.14) implies that the zero solution of (1.1) is US.

Suppose that the zero solution of (1.1) is US. Then for For $\epsilon=1$, there exists a $\delta>0$ such that $\left[n_{0} \geq 0, \phi \in C\left(n_{0}\right),\|\phi\| \leq \delta, n \geq n_{0}\right]$ implies $\left|x\left(n, n_{0}, \phi\right)\right|<1$. Let $m$ be a positive integer and define the sequence of functions $\phi_{m}$ by

$$
\phi_{m}(u)=v a^{-m\left(n_{0}-u\right)} \text { on } 0 \leq u \leq n_{0} .
$$

Let $\psi_{m}(u)=\frac{\delta}{2} v a^{-m\left(n_{0}-u\right)}$ for $0 \leq u \leq n_{0}$. Then, $\left|\psi_{m}(u)\right| \leq \frac{\delta}{2}$. Hence we have $\left|x\left(n, n_{0}, \psi_{m}(s)\right)\right|<\epsilon$. It is clear from (2.15) that $\phi_{m}\left(n_{0}\right)=v$ and 
$\left|\phi_{m}(s)\right| \leq 1$ for $0 \leq s \leq n_{0}$. Thus, from (2.2) we have

$$
\begin{aligned}
\left|R\left(n, n_{0}\right)\right| \frac{\delta}{2} \leq & \left|x\left(n, n_{0}, \psi_{m}\right)\right|+\frac{\delta}{2}\left|\sum_{s=n_{0}}^{n-1} R(n, s+1) \sum_{u=0}^{n_{0}-1} B(s, u) a^{-m\left(n_{0}-u\right)}\right| \\
& +\left|\sum_{s=n_{0}}^{n-1}\right| R(n, s+1)|\lambda(s)| x\left(s, n_{0}, \psi_{m}(s)\right) \mid \\
\leq & 1+\frac{\delta}{2}\left|\sum_{s=n_{0}}^{n-1} R(n, s+1) \sum_{u=0}^{n_{0}-1} B(s, u) a^{-m\left(n_{0}-u\right)}\right| \\
& +\left|\sum_{s=n_{0}}^{n-1}\right| R(n, s+1) \mid \lambda(s) .
\end{aligned}
$$

Now, for fixed $n$,

$$
\left|\sum_{s=n_{0}}^{n-1} R(n, s+1) \sum_{u=0}^{n_{0}-1} B(s, u) a^{-m\left(n_{0}-u\right)}\right| \rightarrow 0 \text { as } m \rightarrow \infty .
$$

By (2.10), there exists a $G>0$ such that $\sum_{s=n_{0}}^{n-1}|R(n, s+1)| \lambda(s) \leq G$. Thus from (2.16)

$$
\left|R\left(n, n_{0}\right)\right| \leq \frac{2}{\delta}(1+G) .
$$

Next, let $\phi \in C\left(n_{0}\right)$ with $\|\phi\|<1$. Define $\psi=\delta \phi$. Then $\|\psi\|<\delta$. Thus, by the definition of $\delta$, we have $\left|x\left(n, n_{0}, \psi\right)\right|<1$ for all $n \geq n_{0}$. It follows from (2.2) and (2.17) that

$$
\begin{aligned}
\left|\sum_{s=n_{0}}^{n-1} R(n, s+1) \sum_{u=0}^{n_{0}-1} B(s, u) \psi(u)\right| \leq & \left|x\left(n, n_{0}, \psi\right)\right|+\left|R\left(n, n_{0}\right)\right|\left|\psi\left(n_{0}\right)\right| \\
& +\sum_{s=n_{0}}^{n-1}|R(n, s+1)| \lambda(s)|| x\left(s, n_{0}, \psi\right) \mid \\
\leq & \left|x\left(n, n_{0}, \psi\right)\right|+\left|R\left(n, n_{0}\right)\right||| \psi\left(n_{0}\right)||+G \\
\leq & 1+2(1+G)+G .
\end{aligned}
$$

Hence,

$$
\left|\sum_{u=0}^{n_{0}-1} \sum_{s=n_{0}}^{n-1} R(n, s+1) \varphi(u)\right| \leq \frac{1}{\delta}\left|\sum_{n=0}^{n_{0}-1} \sum_{s=n_{0}}^{n-1} R(n, s+1) \psi(n)\right| \leq \frac{3}{\delta}(1+G)
$$


for $n \geq n_{0}$ and the proof is complete.

The next Lemma gives necessary and sufficient conditions for the uniform boundedness of $R(n, s)$. Its proof is modeled after Lemma 2.2 of [5].

Lemma 2.1. There exists a positive constant $H$ such that $|R(n, s)| \leq H$ for $n \geq s \geq 0$ if and only if

$$
\sup _{n \geq s \geq 0}\left|\sum_{u=s}^{n-1} R(n, u+1)\left(D(u)+\sum_{v=s}^{u} R(n, u+1) B(u, v)\right)\right|<\infty .
$$

Proof. By solving equation (1.4) for $\triangle_{s} R(n, s)$, and summing it from $s$ to $n-1$ and then changing the order of summations, we arrive at

$$
\begin{aligned}
R(n, s) & =I+\sum_{u=s}^{n-1} R(n, u+1)[A(u)-I]+\sum_{v=s}^{n-1} \sum_{u=v}^{n-1} R(n, u+1) B(u, v) \\
& =I+\sum_{u=s}^{n-1} R(n, u+1) D(u)+\sum_{u=s}^{n-1} \sum_{v=s}^{u} R(n, u+1) B(u, v) \\
& =I+\sum_{u=s}^{n-1} R(n, u+1)\left[D(u)+\sum_{v=s}^{u} B(u, v)\right] .
\end{aligned}
$$

Hence, the result follows.

For Theorem 2.4, we assume

$$
\sup _{n \geq n_{0} \geq 0} \sum_{u=0}^{n_{0}-1}\left|\sum_{s=n_{0}}^{n} R(n, s+1) B(s, u)\right|<+\infty .
$$

Theorem 2.3. Suppose that $|R(n, s+1)| \leq H(s)$ for $0 \leq s \leq n<\infty$ with

$$
\sup _{n \geq 0} \sum_{s=0}^{n-1} H(s) \lambda(s) \leq K \text { for } K>0 .
$$

Then, the zero solution of (1.1) is US if and only if (2.7) holds .

Proof. If (2.7) holds, then for $0 \leq n_{0} \leq s \leq n$, we have $|R(n, s+1)| \leq$ $H(s)<\infty$. From (2.13), we obtain 


$$
\begin{aligned}
|x(n)| \leq & \left\{\left|R\left(n, n_{0}\right)\right|+\sum_{u=0}^{n_{0}-1}\left|\sum_{s=n_{0}}^{n-1} R(n, s+1) B(s, u)\right|\right\}\|\phi\| \\
& +\sum_{s=n_{0}}^{n-1}|R(n, s+1)| \lambda(s)|x(s)| \\
\leq & E\|\phi\|+\sum_{s=n_{0}}^{n-1} H(s) \lambda(s)|x(s)| .
\end{aligned}
$$

Applying the discrete Gronwall's inequality, see [9], we get

$$
|x(n)| \leq E\|\phi\| \exp \left(\sum_{s=n_{0}}^{n-1} H(s) \lambda(s)\right) \leq E\|\phi\| \exp (K):=L\|\phi\|
$$

This proves that the zero solution of (1.1) is US. The proof of the converse of this theorem is similar to the proof of the converse of Theorem 2.2.

THEOREM 2.4 .

(i) If (2.7)-(2.9) and (2.18)-(2.19) hold, then the zero solution of (1.1) is US.

(ii) If the zero solution of (1.1) is US and (2.10) holds, then (2.18)- (2.19) hold.

Proof. Conditions (2.18)-(2.19) hold if and only if (2.7) holds. Therefore the results follow directly from Theorem 2.2 .

THEOREM 2.5.

i. Suppose that (2.6)-(2.9), and

$$
\left\{\left|R\left(n, n_{0}\right)\right|+\sum_{u=0}^{n_{0}-1}\left|\sum_{s=n_{0}}^{n-1} R(n, s+1) B(s, u)\right|\right\} \rightarrow 0
$$

as $n-n_{0} \rightarrow+\infty$ uniformly,

$$
\sum_{s=n_{0}}^{n-1}|R(n, s+1)| \rightarrow 0
$$

as $n-n_{0} \rightarrow+\infty$ uniformly hold. Then, the zero solution of (1.1) is UAS. ii. If the zero solution of (1.1) is UAS and (2.23) hold, then (2.22) holds. 
Proof. Suppose that (2.6)-(2.9) hold. Then, by Theorem 2.2, the zero solution is obviously US. Let $B_{1}>0$ be given and $\phi \in C\left(n_{0}\right)$ on $0 \leq s \leq n_{0}$ with $\|\phi\| \leq B_{1}$. Then, it follows from (2.13) and (2.14) that,

$$
\begin{aligned}
|x(n)| \leq & \left\{\left|R\left(n, n_{0}\right)\right|+\sum_{u=0}^{n_{0}-1}\left|\sum_{s=n_{0}}^{n-1} R(n, s+1) B(s, u)\right|\right\}\|\phi\| \\
& +\sum_{s=n_{0}}^{n-1}|R(n, s+1)| N J \| \phi|| \\
\leq & {\left[\left|R\left(n, n_{0}\right)\right|+\sum_{u=0}^{n_{0}-1}\left|\sum_{s=n_{0}}^{n-1} R(n, s+1) B(s, u)\right|\right] B_{1} } \\
& +\left[N J \sum_{s=n_{0}}^{n-1}|R(n, s+1)|\right] B_{1} .
\end{aligned}
$$

From (2.22) and (2.23), it follows that for any $\epsilon>0$, there exists a constant $T>0$ such that

$$
\left[\left|R\left(n, n_{0}\right)\right|+\sum_{u=0}^{n_{0}-1}\left|\sum_{s=n_{0}}^{n-1} R(n, s+1) B(s, u)\right|+N J \sum_{s=n_{0}}^{n-1}|R(n, s+1)|\right]<\frac{\epsilon}{B_{1}}
$$

for all $n \geq T+n_{0}$. Thus, $|x(n)|<\epsilon$ for all $n \geq T+n_{0}$. This implies that the zero solution of (1.1) is UAS.

Conversely, suppose that the zero solution of (1.1) is UAS. Then it is US. Let $\phi \in C\left(n_{0}\right)$ with $\|\phi\| \leq 1$. Then, for any $\epsilon>0$, there exists $T>0$ such that $\left|x\left(n, n_{0}, \phi\right)\right|<\epsilon$ for $n \geq T+n_{0}$. By making use of (2.16) and by the argument of Theorem 2.2 (ii), we have $\left|R\left(n, n_{0}\right)\right|<\epsilon$ for all $n \geq T+n_{0}$. Now using (2.13) in (2.2), we get

$$
\begin{aligned}
& \left|\sum_{u=0}^{n_{0}-1}\left(\sum_{s=n_{0}}^{n-1} R(n, s+1) B(s, u)\right) \phi(u)\right| \\
\leq & \left|x\left(n, n_{0}, \phi\right)\right|+\left|R\left(n, n_{0}\right)\right|+N J \sum_{s=n_{0}}^{n-1}|R(n, s+1)|<3 \epsilon
\end{aligned}
$$

for all $n \geq T+n_{0}$. This implies

$$
\sum_{u=0}^{n_{0}-1}\left|\sum_{s=n_{0}}^{n-1} R(n, s+1) B(s, u)\right|<3 \epsilon
$$


for all $n \geq T+n_{0}$. This shows that (2.22) hold and the proof is complete.

REMARK 2.1. The function

$$
|R(n, s)|+\sum_{u=s}^{n-1}\left|\sum_{v=0}^{s-1} R(n, u+1) B(u, v)\right|
$$

can serve as a Liapunov functional to directly verify conditions (2.7) and (2.22)-(2.23). (See Example 3.1).

Lemma 2.2. The resolvent $R(n, s) \rightarrow 0$ as $n-s \rightarrow+\infty$ uniformly if and only if

$$
\left\{I+\sum_{u=s}^{u=n-1}|R(n, u)|\left(D(u)+\sum_{v=s}^{u} B(u, v) \mid\right)\right\} \rightarrow 0
$$

as $n-s \rightarrow+\infty$ uniformly.

The proof follows directly from Lemma 2.1.

THEOREM 2.6 .

(i) If (2.6)-(2.9), (2.23)-(2.25), and

$$
\sum_{u=0}^{n_{0}-1}\left|\sum_{s=n_{0}}^{n-1} R(n, s+1) B(s, u)\right| \rightarrow 0
$$

as $n-n_{0} \rightarrow+\infty$ uniformly hold, then the zero solution of (1.1) is UAS.

The proof of Theorem 2.6 follows directly from Lemma 2.2 and Theorem 2.5.

Using (2.21) of Theorem 2.3, we obtain the following theorem which is more practical when the sum of $R(n, s+1)$ with $A(n)$ and $\sum_{s=0}^{n} B(n, s)$ can be estimated.

Theorem 2.7. Suppose that (2.7) and (2.20) hold. Then, the zero solution of (1.1) is UAS if and only if (2.22) and

$$
\sum_{s=0}^{n-1}|R(n, s+1)| \lambda(s) \rightarrow 0 \text { for } n-n_{0} \rightarrow+\infty \text { uniformly }
$$

hold.

Proof. Suppose that (2.7) and (2.20) hold. Then, by Theorem 2.3, the zero 
solution is US.

Let $B_{1}>0$ be a given constant and $\phi \in C\left(n_{0}\right)$ on $0 \leq s \leq n_{0}$ with $\|\phi\|<B_{1}$. Using (2.21) in (2.2), we obtain

$$
\begin{aligned}
|x(n)| \leq & \left\{\left|R\left(n, n_{0}\right)\right|+\sum_{u=0}^{n_{0}-1}\left(\sum_{s=n_{0}}^{n-1} R(n, s+1) B(s, u)\right)\right\}\|\phi\| \\
& +L \sum_{s=n_{0}}^{n-1}|R(n, s+1)| \lambda(s)\|\phi\| .
\end{aligned}
$$

Applying (2.22) and (2.27) in the above inequality gives the UAS. The converse of this theorem follows from the proof of the converse of Theorem 2.5.

\section{SCALAR EQUATION}

We end the paper by furnishing an example in which we show that the zero solution of the scalar nonlinear volterra discrete equation

$$
x(n+1)=a(n) x(n)+\sum_{s=0}^{n} b(n, s) x(s)+g(n, x(n))
$$

where $|g(n, x(n))| \leq \lambda(n)|x(n)|$, is UAS.

EXAmPle 3.1. Consider equation (3.1) and suppose there are positive constants $\gamma, h, B$ and $K$ with $K<1$ satisfying the following conditions for $t \geq 0$ :

(i) $\quad-a(n)+K\left(1-\sum_{s=0}^{n}|b(n, s)|\right)>0$,

(ii) For each $\gamma>0$, there exists $h>0$ such that $\sum_{s=n}^{n+h-1}|a(s)| \geq \gamma$,

(iii) $\frac{1}{|a(n)|} \sum_{s=0}^{n_{0}}|b(n, s)| \rightarrow 0$ as $n-n_{0} \rightarrow+\infty$ uniformly on $\{n \mid a(n) \neq 0\}$,

(iv) $\sum_{s=0}^{n_{0}-1}|b(n, s)| \geq \lambda(n)$ for $n_{0} \geq 0$, 
(v) $\sum_{0}^{n} \lambda(s)<\infty$ for all $n \geq 0$, and

(vi) $\sum_{u=0}^{\infty}|b(u, s)| \leq B$.

Then the zero solution of (3.1) is UAS.

Proof. Define the discrete Liapunov functional, see [5], $V(s)$ on $[0, n-1]$ by

$$
V(s)=|R(n, s)|+\sum_{u=s}^{n-1} \sum_{v=0}^{s-1}|R(n, u+1)||b(u, v)|
$$

where $R(n, s)$ is the resolvent of (3.1) with $g=0$, satisfying

$$
R(n, s+1) a(s)+\sum_{u=s}^{n-1} R(n, u+1) b(u, s)-R(n, s)=0, \quad R(n, n)=1 .
$$

Then using (i) we have

$$
\begin{aligned}
\triangle V(s)= & |R(n, s+1)|-|R(n, s)| \\
& +\sum_{u=s+1}^{n-1} \sum_{v=0}^{s}|R(n, u+1)||b(u, v)|-\sum_{u=s}^{n-1} \sum_{v=0}^{s-1}|R(n, u+1)||b(u, v)| \\
\geq & (-|a(s)|+1)|R(n, s+1)| \\
& -|R(n, s+1)||b(s, s)|-|R(n, s+1)| \sum_{v=0}^{s-1}|b(s, v)| \\
= & \left(1-|a(s)|-\sum_{v=0}^{s}|b(s, v)|\right)|R(n, s+1)| \\
\geq & \left(\frac{1}{K}-1\right)|a(s)||R(n, s+1)| .
\end{aligned}
$$

So we have, $\triangle V(s)>0$.

This yields that for $n \geq n_{0} \geq 0, V\left(n_{0}\right) \leq V(n)=|R(n, n)|=1$. That is,

$$
\left|R\left(n, n_{0}\right)\right|+\sum_{u=n_{0}}^{n-1} \sum_{v=0}^{n_{0}-1}|R(n, u+1)||b(u, v)| \leq 1 .
$$

Hence (2.7) is satisfied.

By summing (3.2) from 0 to $n-1$, we obtain

$$
\left(\frac{1}{K}-1\right) \sum_{s=0}^{n-1}|a(s)||R(n, s+1)| \leq V(n)-V(0) \leq 1
$$


or

$$
\sum_{s=0}^{n-1}|a(s)||R(n, s+1)| \leq \frac{K}{1-K}=: D .
$$

Note that (3.3) implies that there exists a constant $H>0$ such that

$$
\sup _{s \geq n_{0} \geq 0}|R(n, s+1)|=H \leq 1 \text { for } 0 \leq s \leq n<\infty .
$$

Hence, by (v) we have

$$
\sup _{n \geq 1} \sum_{s=0}^{n-1} H \lambda(s)<\infty
$$

Thus condition (2.20) is satisfied and by Theorem 2.3, the zero solution of (3.1) is US. By (iii), for any $\epsilon>0$ there exists $N_{1}>0$ such that for $u \geq N_{1}+s-1$ implies

$$
\sum_{v=0}^{s-1}|b(u, v)| \leq \frac{\epsilon}{(3+B) D}|a(u)| .
$$

Thus, for $n \geq N_{1}+s-1$ we have

$$
\begin{aligned}
& \sum_{u=s}^{s+N_{1}-1}|R(n, u+1)| \sum_{v=0}^{s-1}|b(u, v)| \\
= & \sum_{u=s}^{n-1}|R(n, u+1)| \sum_{v=0}^{s-1}|b(u, v)|+\sum_{u=s+N_{1}}^{n-1}|R(n, u+1)| \sum_{v=0}^{s-1}|b(u, v)| \\
\leq & \frac{\epsilon}{(3+B) D} \sum_{u=s+N_{1}}^{n-1}|R(n, u+1)||a(u)|+\sum_{u=s}^{s+N_{1}-1}|R(n, u+1)| \sum_{v=0}^{s-1}|b(u, v)| \\
\leq & \frac{\epsilon}{3+B}+\sum_{u=s}^{s+N_{1}-1}|R(n, u+1)| \sum_{v=0}^{s-1}|b(u, v)| .
\end{aligned}
$$

Let $\beta=\frac{K}{1-K}$ and $\alpha=\frac{3+B}{\epsilon \beta}$. By (ii), there exists an $h>0$ such that $\sum_{v=s}^{s+h-1}|a(v)| \geq \alpha$, and

$$
\left|R\left(n, n_{s}+1\right)\right| \beta \sum_{v=s}^{s+h-1}|a(v)| \leq \beta \sum_{u=s}^{s+h-1}|R(n, u+1)||a(u)|,
$$

for $n_{s} \in[s, s+h-1]$ and $n \geq s+h$, where

$$
\left|R\left(n, n_{s}+1\right)\right|=\min _{s \leq u \leq s+h-1}|R(n, u+1)| .
$$




\section{TOUHID M. KAHANDAKER AND YOUSSEF N. RAFFOUL}

Using (3.4) in the above inequality we arrive at

$$
\left|R\left(n, n_{s}+1\right)\right| \leq \frac{1}{\beta \sum_{v=s}^{s+h-1}|a(v)|}<\frac{\epsilon}{3+B} .
$$

Choose $N>1$ so that $\frac{\beta N \epsilon}{3+B}>1$. For each $n_{0} \geq 0$ and $n \geq n_{0}+(N+1)\left(N_{1}+\right.$ $h-1)$, define $\left\{n_{j}\right\}$ with

$$
n(j-1)+N_{1} \leq n_{j} \leq n(j-1)+N_{1}+h-1, \quad j=1,2,3 \ldots . N
$$

such that

$$
\left|R\left(n, n_{j}+1\right)\right|<\frac{\epsilon}{3+B} .
$$

It follows that $n_{N} \leq n_{0}+N\left(N_{1}+h-1\right)$ and by (3.4) we arrive at

$$
\sum_{j=1}^{N}\left(\sum_{u=n_{j}}^{n_{j}+N_{1}-1} \beta|R(n, u+1)||a(u)|\right) \leq \sum_{u=n_{0}}^{n-1} \beta|R(n, u+1)||a(u)| \leq 1 .
$$

Since $\frac{\beta N \epsilon}{3+B}>1$, it follows from the above inequality there exists $n_{k}, 1 \leq k \leq$ $N$ such that

$$
N \sum_{u=n_{k}}^{n_{k}+N_{1}-1} \beta|R(n, u+1) \| a(u)| \leq 1
$$

Or,

$$
\sum_{u=n_{k}}^{n_{k}+N_{1}-1}|R(n, u+1)||a(u)|<\frac{\epsilon}{3+B} .
$$

Since $V(s)$ is increasing, we have

$$
V\left(n_{k}\right) \leq V\left(n_{k}+1\right) .
$$

Hence, using (3.5)-(3.6) and (vi) we arrive at

$$
\begin{aligned}
& \left|R\left(n, n_{k}\right)\right|+\sum_{u=n_{k}}^{n-1} \sum_{v=0}^{n_{k}-1}|R(n, u+1)||b(u, v)| \\
\leq & \left|R\left(n, n_{k}+1\right)\right|+\sum_{u=n_{k}}^{n-1} \sum_{v=0}^{n_{k}-1}|R(n, u+1)||b(u, v)| \\
& +\sum_{u=n_{k}+1}^{n-1}|R(n, u+1)|\left|b\left(u, n_{k}\right)\right| \\
\leq & \frac{\epsilon}{3+B}+\frac{2 \epsilon}{3+B}+\frac{B \epsilon}{3+B}=\epsilon .
\end{aligned}
$$


This yields

$$
\left|R\left(n, n_{0}\right)\right|+\sum_{u=n_{0}}^{n-1} \sum_{v=0}^{n_{0}-1}|R(n, u+1)||b(u, v)|=V\left(n_{0}\right) \leq V\left(n_{k}\right)<\epsilon
$$

for $n \geq n_{0}+(N+1)\left(N_{1}+h-1\right) \geq n_{k}, N>\frac{3+B}{\beta \epsilon}$. Hence condition (2.22) is satisfied. Next, for $n \geq n_{0}+(N+1)\left(N_{1}+h-1\right) \geq n_{k}$ we have by using condition (iv) in (3.8).

$$
\begin{aligned}
\left|R\left(n, n_{0}\right)\right| & +\sum_{u=n_{0}}^{n-1}|R(n, u+1)| \lambda(u) \\
& \leq\left|R\left(n, n_{0}\right)\right|+\sum_{u=n_{0}}^{n-1}|R(n, u+1)| \sum_{v=0}^{n_{0}-1}|b(u, v)| \\
& \leq\left|R\left(n, n_{0}\right)\right|+\sum_{u=n_{0}}^{n-1} \sum_{v=0}^{n_{0}-1}|R(n, u+1)||b(u, v)| \\
& <\varepsilon .
\end{aligned}
$$

Hence, (2.27) follow directly from (3.9) and the zero solution of (3.1) is UAS by Theorem 2.7 .

\section{References}

[1] Agarwal, R., and Pang, P. Y., On a generalized difference system, Nonlinear Anal., TM and Appl., 30(1997), 365-376.

[2] Crisci, M. R., Kolmanovskii, V. B., and Vecchio. A., Boundedness of discrete Volterra equations, J. Math. Analy. Appl. 211(1997), 106-130.

[3] Elaydi, S., Periodicity and stability of linear Volterra difference systems, J. Math. Analy. Appl. 181(1994), 483-492.

[4] Elaydi, S., and Murakami, S., Uniform asymptotic stability in linear Volterra difference equations, Journal of Difference Equations 3(1998), 203-218.

[5] Eloe, P., Islam, M., and Raffoul, Y., Uniform asymptotic stability in nonlinear Volterra discrete systems, Computers and Mathematics with applications, Special Issue Advances in Difference Equations IV, to appear. 
[6] Islam, M., and Raffoul, Y., Uniform asymptotic stability in linear Volterra difference equations, PanAmerican Mathematical Journal, to appear.

[7] Islam, M., and Raffoul, Y., Stability properties of linear Volterra integrodifferential equations with nonlinear perturbation, Communication of Applied Analysis, to appear.

[8] Kelly, W., and Peterson, A., Difference equations an introduction with applications, Academic Press, New York, 2000.

[9] Lakshmikantham, L., and Trigiante, D., Theory of difference equations:Numerical methods and applications, Academic Press, New York, 1991.

[10] Medina, R., Asyptotic behavior of Volterra difference equations, Computers and Mathematics with Applications, to appear.

[11] Medina, R., The asyptotic behavior of the solutions of a Volterra difference equation, Computers Math. Appl. 181(1994), 19-26.

[12] Medina, R., Solvability of discrete Volterra equations in weighted spaces, Dynamic Systems and Appl. 5(1996), 407-422.

[13] Raffoul, Y., Boundedness and periodicity of Volterra systems of difference equations, Journal of Difference Equations and Applications 4(1998), 381-393. 